\title{
Cardiovascular magnetic resonance imaging in valvular heart disease
}

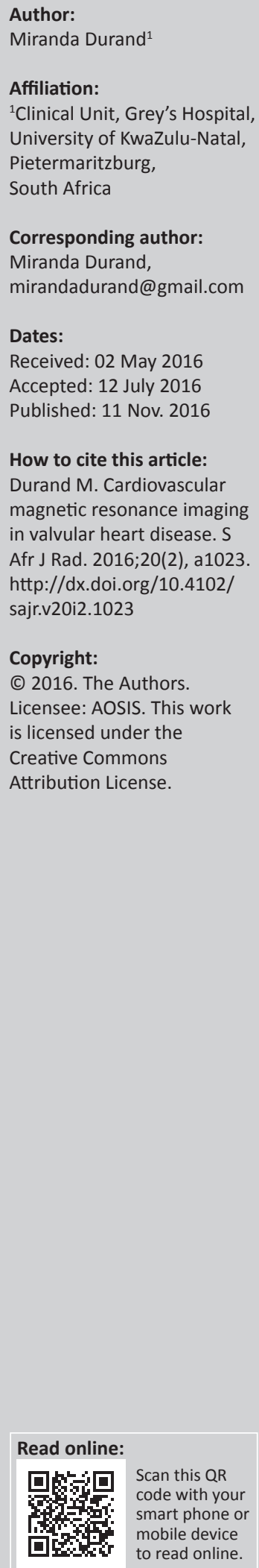

The superb spatial and temporal resolution of cardiovascular magnetic resonance (CMR), as well as its reproducibility and independence from patient body habitus and acoustic window, make it an excellent tool for not only diagnostic assessment but also follow-up of valvular heart disease (VHD) patients to determine the optimal time for intervention. This article reviews the anatomy and pulse sequences used to assess these patients and provides an approach for CMR valvular assessment. In addition, it reviews the role of CMR in the assessment of patients with VHD.

\section{Introduction}

Echocardiography remains the first line of imaging assessment for valvular heart disease (VHD) because of its wide availability, portability and low cost. Recent developments in real-time 3D echocardiography have improved the imaging of native valve anatomy and the quantification of valve geometry and function. ${ }^{1}$ Echocardiography, however, is often limited by the patient's acoustic window. Also, inter-observer variation limits the assessment of changes in disease severity and progression.

In 2006, the American College of Cardiology acknowledged the increasing role that cardiovascular magnetic resonance (CMR) has to play in the assessment of patients with VHD, and gave CMR an 'A' appropriateness rating for 'characterization of native and prosthetic cardiac valves - including planimetry of stenotic disease and quantification of regurgitation diseases' and 'patient(s) with technically limited images from echocardiograms or TEE'.2

The superb spatial and temporal resolution of CMR, as well as its reproducibility and independence from patient body habitus and acoustic window, make it an excellent tool for not only diagnostic assessment but also follow-up of these patients to determine the optimal time for intervention. ${ }^{3}$

The present article reviews the anatomy and pulse sequences used to assess patients with VHD and provides an approach for CMR valvular assessment.

\section{Pulse sequences}

For patients with VHD, start with a basic CMR protocol:

- axial black blood images of the thorax

- two-chamber cine steady state free precession (SSFP) images

- four-chamber cine SSFP images

- left ventricular outflow tract (LVOT) cine SSFP images

- short-axis cine SSFP images of the ventricles

- axial cine SSFP images through the valve.

After this, the protocol is tailored to the valve involved. For left-sided heart valves, phase contrast images should be added through the aorta. For right-sided heart valves, right ventricular outflow tract (RVOT) cine SSFP images and phase contrast images are added through the pulmonary artery.

When determining if the stenosis or pathology is at the level of the valve or subvalvular, as in cases involving the aorta and pulmonary valves, coronal images through the valve may also be helpful (Figure 1).

The two-chamber, four-chamber and LVOT cine images are useful to assess for mitral regurgitation. The axial cine images though the mitral valve give a good view of all the leaflets, allowing assessment of leaflet morphology. 


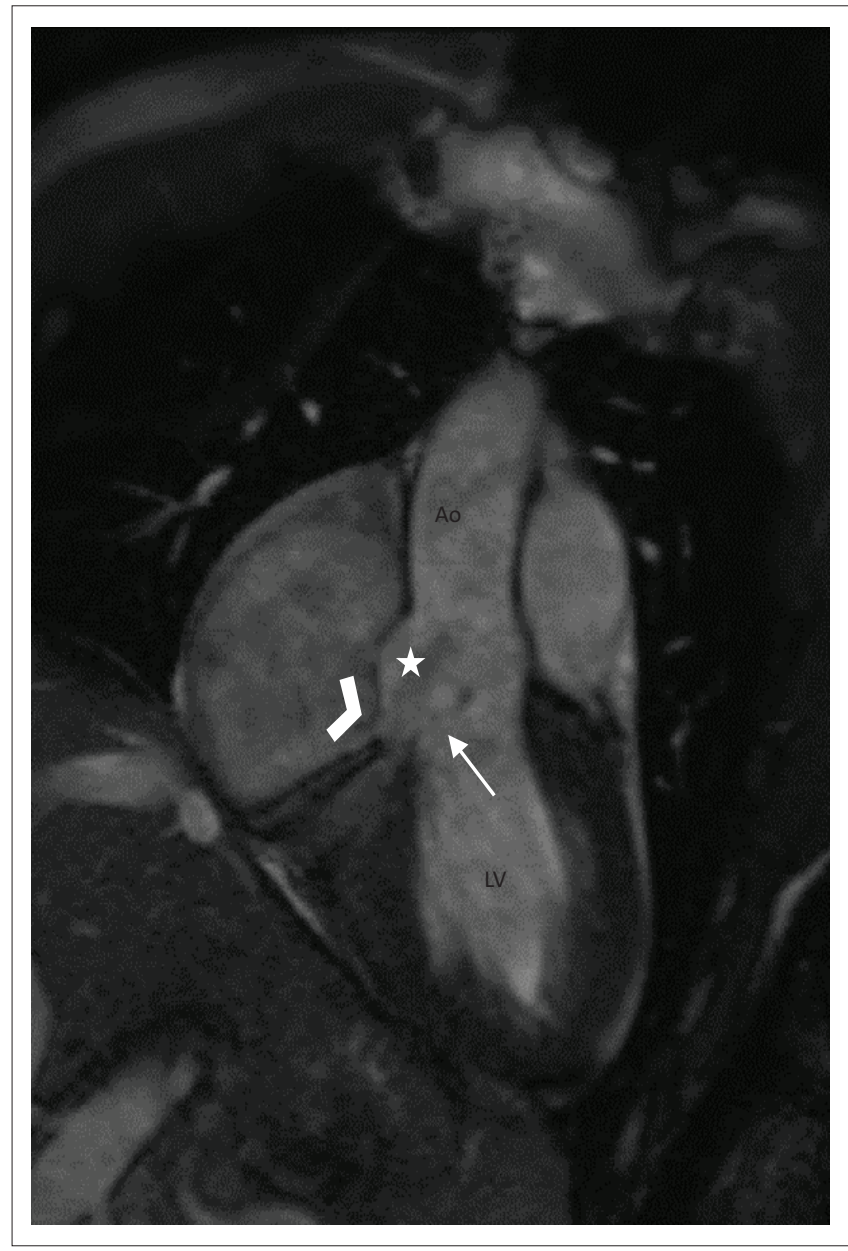

Ao, aorta; LV, left ventricle.

FIGURE 1: Coronal SSFP image of the aortic valve demonstrates an aneurysm (arrowhead) of the sinus of Valsalva (*). The coronal image demonstrates the aneurysm above the level of the leaflet (white arrow). Cine image available at https://www.youtube.com/watch?v=mHb_6cMIHMQ\&feature= youtu.be
Similarly, the four-chamber and axial cine images are useful to assess for tricuspid regurgitation. The fourchamber cine image also best depicts the attachment of the septal leaflet of the tricuspid valve for the assessment of Ebstein's abnormality. For assessing leaflet morphology, the axial cine images through the tricuspid valve are most useful.

\section{Cardiovascular magnetic resonance in valvular heart disease}

The assessment of valves using CMR can be structured in the following way:

- normal anatomy

- valve morphology

- valve function

- ventricular function.

\section{Anatomy}

\section{Aortic valve}

The aortic valve is a tricuspid valve with three valve cusps: the right coronary cusp, the left coronary cusp and the noncoronary cusp (Figure 2a). Small spaces are seen between the attachments of the cusps to the aortic wall, called commissures (Figure 2b). On the coronal images of the aortic root the aortic cusps, the sinus of Valsalva and sinotubular junction can be assessed in plane (Figure 2c). The coronary arteries normally arise from the sinus of Valsalva below the level of the sinotubular junction.

\section{Mitral valve}

The mitral valve consists of leaflets (the anterior and posterior leaflets; Figure 3a), each of which can be divided into three
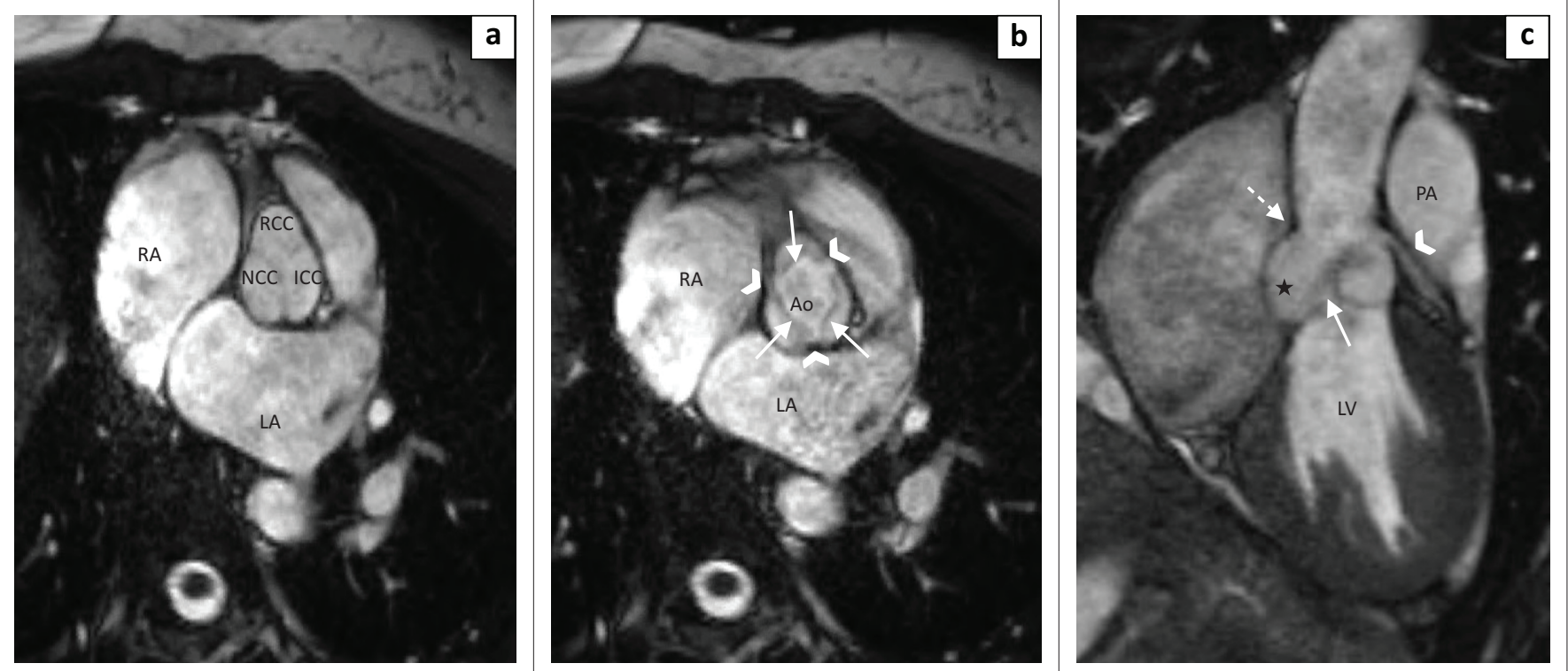

FIGURE 2: (a) Axial cine SSFP image through the aortic valve in diastole demonstrates a closed valve with three valve cusps: the right coronary cusp (RCC), the left coronary cusp (LCC) and non-coronary cusp (NCC). The left atrium (LA) and right atrium (RA) are also identified; (b) Axial cine SSFP image through the aortic valve in systole demonstrates an open valve with the cusp leaflets (white arrows) and the commissures between the cusp attachments (arrowheads). The aorta (Ao), left atrium (LA) and right atrium (RA) are identified. (c) Coronal SSFP cine image of the aortic valve demonstrates the aortic cusp (white arrow), the coronary sinus (*) and the sinotubular junction (dashed arrow) and the coronary artery coming off the coronary sinus (arrowhead). The left ventricle (LV) and pulmonary artery (PA) are identified. 


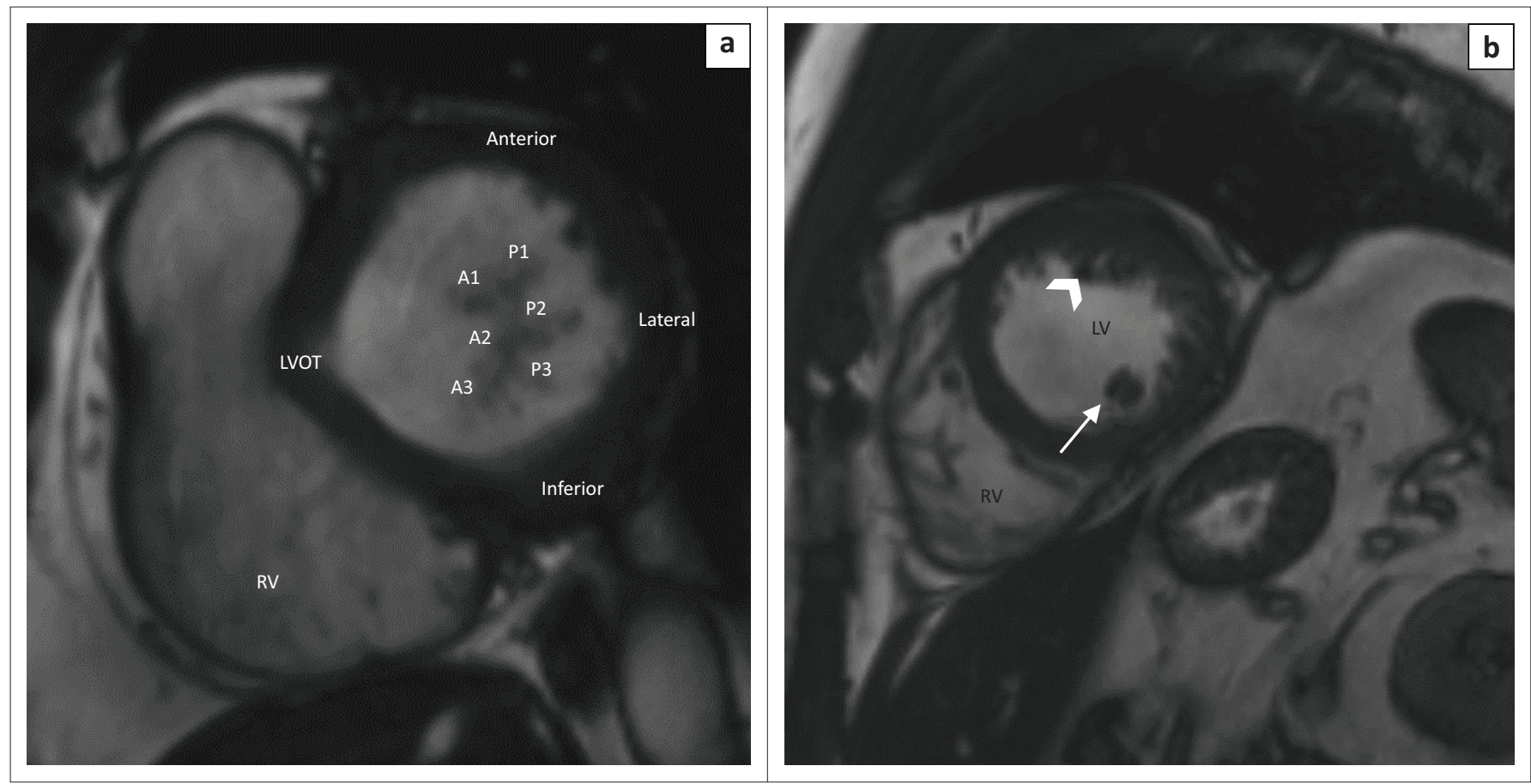

LVOT, left ventricular outflow tract; RV, right ventricle; RV, right ventricle; LV, left ventricle.

FIGURE 3: (a) Short-axis cine SSFP image demonstrates a closed mitral valve during systole, the anterior leaflet with A1, A2 and A3 portions, as well as the posterior leaflet with P1, P2 and P3 segments. (b) Short-axis cine SSFP image demonstrates the anterior lateral (arrowhead) and inferior medial (white arrow) papillary muscles of the mitral valve.

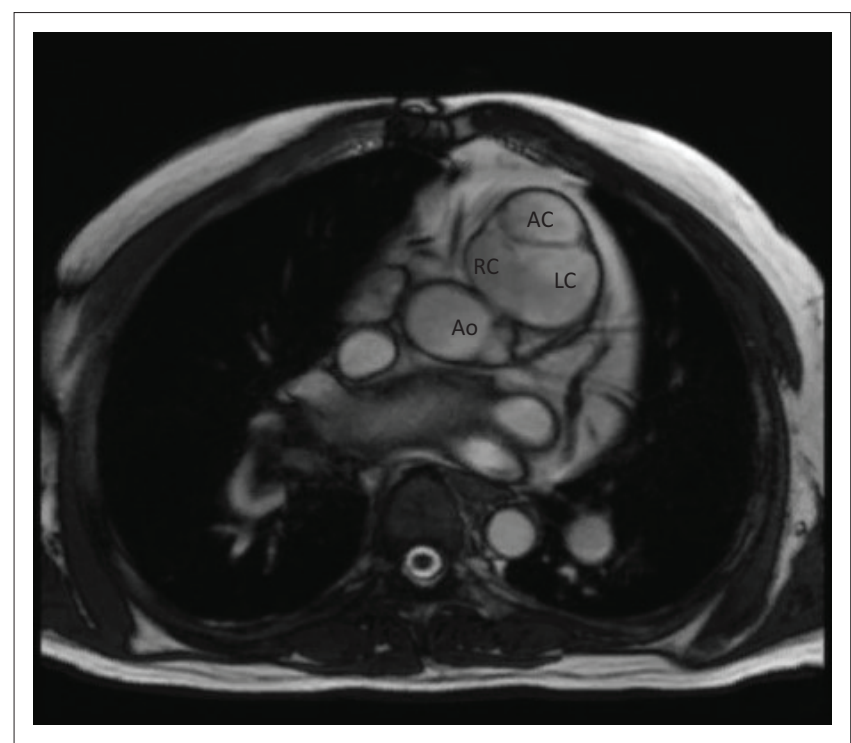

Ao, aorta.

FIGURE 4: Axial cine SSFP image through the pulmonary valve demonstrates the three cusps of the pulmonary valve: the anterior (AC), right (RC) and left (LC) cusps.

components. Each of the leaflets are attached to a papillary muscle via a tendinous cord (Figure $3 b$ ), and two sets of papillary muscles are seen on the short-axis images - the anterior lateral and the inferior medial papillary muscles.

\section{Pulmonary valve}

The pulmonary artery is the most anterior great vessel on the axial images. The pulmonary valve appears anatomically very similar to the aortic valve and also has three cusps: the anterior, left and right cusps (Figure 4).

\section{Tricuspid valve}

The tricuspid valve is a three-leaflet valve with an anterior, septal and posterior leaflet (Figure 5a). Each of these leaflets is attached to similarly named papillary muscles via tendinous cords (Figure 5b).

\section{Valve morphology}

\section{Number of leaflets}

Part of the morphological assessment of the cardiac valves is to assess the number of valve leaflets. Congenital leaflet number abnormalities are fairly uncommon and are most often in the aortic valve.

The most common leaflet abnormality of the aortic valve is a bicuspid aortic valve. The most common form of the bicuspid aortic valve is that of a valve with one raphe (Figure 6a) which is the result of fusion of two adjacent cusps. ${ }^{4}$ On rare occasions, the bicuspid valve is secondary to a bisinus valve, with only two sinuses of Valsalva and two cusps. No raphes are seen in these cases but the abnormality still results in the same characteristic 'fish mouth' opening of the valve (Figure $6 b$ ).

Leaflet number abnormalities in the other valves are extremely rare, but leaflets ranging from 2 to 5 have been described for the pulmonary and tricuspid valves. ${ }^{5}$

\section{Attachment abnormalities}

The most common attachment abnormality of the valves is Ebstein's abnormality, where there is apical displacement of the implantation of the septal leaflet of the tricuspid 

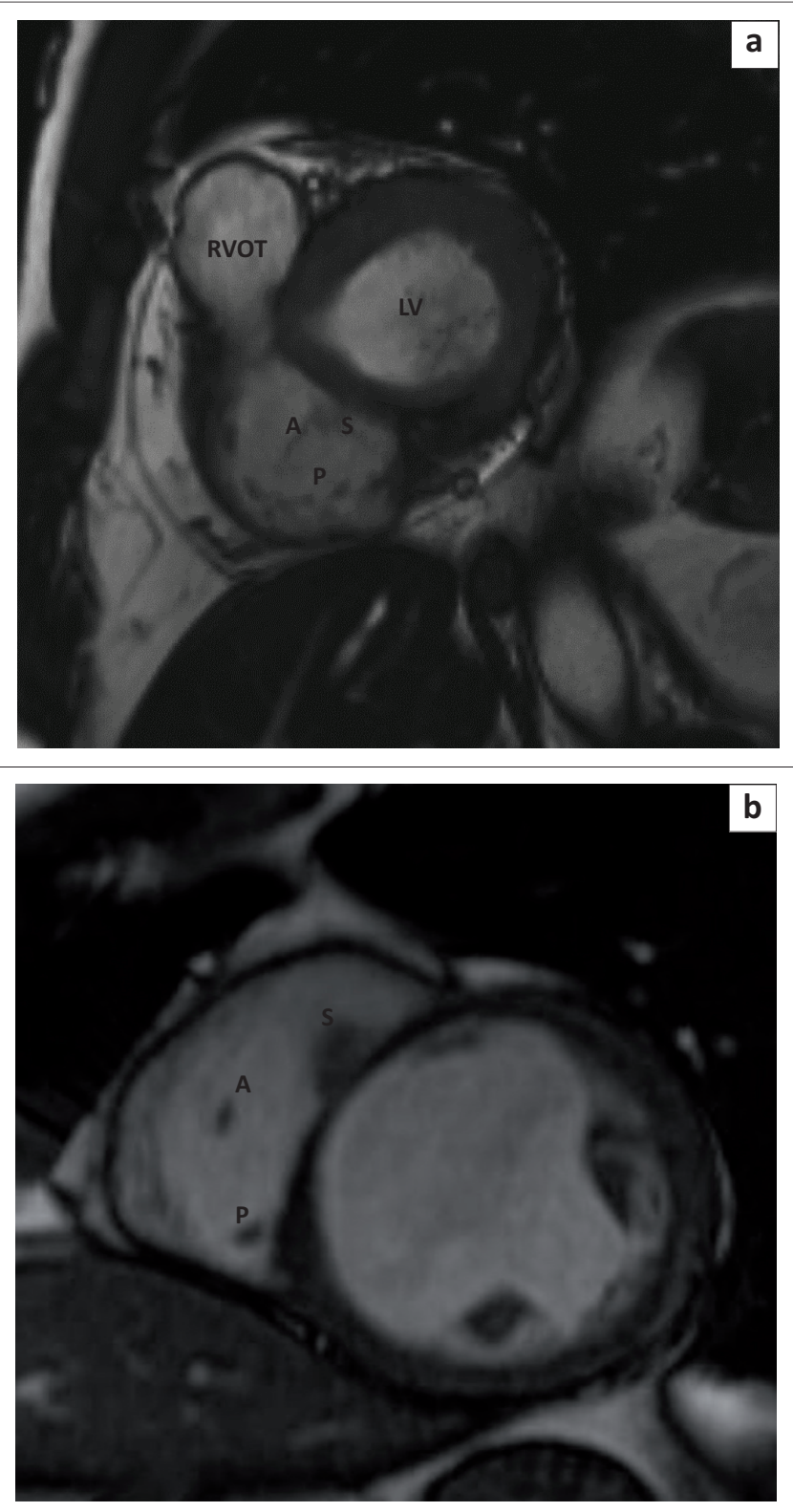

FIGURE 5: (a) Short-axis cine SSFP image of the tricuspid valve demonstrates the anterior (A), septal (S) and posterior (P) leaflets. The left ventricle (LV) and right ventricular outflow tract (RVOT) are identified; (b) Short-axis cine SSFP image demonstrates the anterior (A), septal (S) and posterior (P) papillary muscles of the tricuspid valve.

valve (Figure 7). The degree of apical displacement varies with Ebstein's abnormality and is classified as abnormal if apical displacement of the septal leaflet of more than $8 \mathrm{~mm} / \mathrm{m}^{2}$ is present. ${ }^{6}$ This results in part of the right ventricle being incorporated into the right atrium and subsequently increases the right atrial size and decreases the functional right ventricle volume.

\section{Leaflet integrity}

The leaflets need to be assessed for tears, clefts or prolapses. Normally the flow jet from regurgitation secondary to ventricular dilatation is directed directly posteriorly. Tears and clefts can be acquired or congenital and are normally seen as an extra or eccentric flow jet arising from the leaflet (Figure 8).
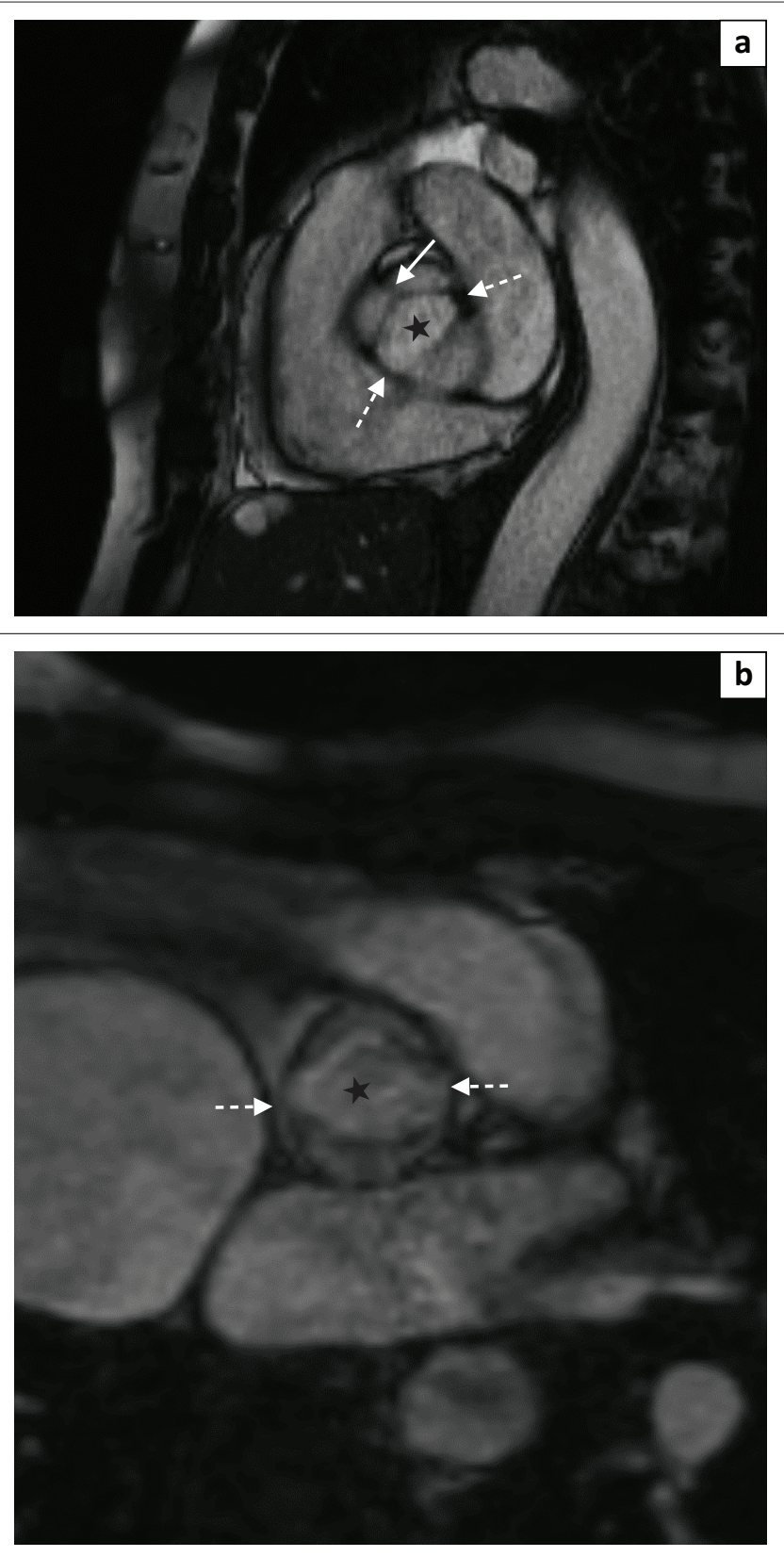

FIGURE 6: (a) Short-axis cine image of the aortic valve demonstrates the very characteristic 'fish mouth' opening of the bicuspid aortic valve secondary to a raphe (solid white arrow). Two normal commissures can also be seen (dashed white arrows). Cine image available at https://www.youtube.com/ watch?v=sWx3Jlax4DQ\&feature=youtu.be; (b) Axial cine SSFP image of the aortic valve demonstrates only two coronary sinuses of Valsalva with two normal commissures (dashed white arrows) resulting in the characteristic 'fish mouth' opening of the bicuspid aortic valve. Cine image available at https:// www.youtube.com/watch?v=1NHgzOb-Y_w\&feature=youtu.be

Prolapse is seen as atrial displacement of the leaflets. The easiest way to assess this is to draw a line through the annulus and measure the distance from the line to the leaflet. If the displacement is more than $2 \mathrm{~mm}$, it is in keeping with prolapse. ${ }^{7}$ An associated regurgitation jet is also normally seen.

With leaflet prolapse, as with tears and clefts, the flow jet is eccentric. In mitral valve prolapse, the jet is directed anteromedially towards the septum with prolapse of the posterior leaflet, and is postero-central or postero-lateral with anterior leaflet prolapse. In the instance of bi-leaflet prolapse, the flow jet is in a postero-medial direction (Figure 9). ${ }^{8}$ 


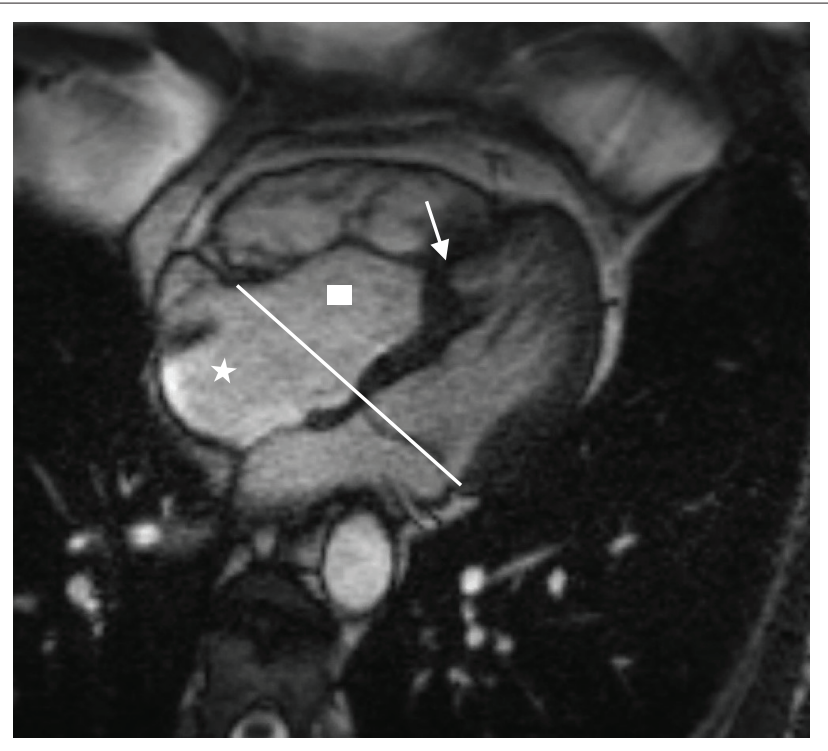

FIGURE 7: Four-chamber SSFP cine image demonstrates the apical displacement of the septal leaflet of the tricuspid valve (white arrow) from the level of the mitral valve annulus (white line). This results in a true right atrium (*) and an atrialised portion of the right ventricle (white square), and a reduced functional right ventricle volume.

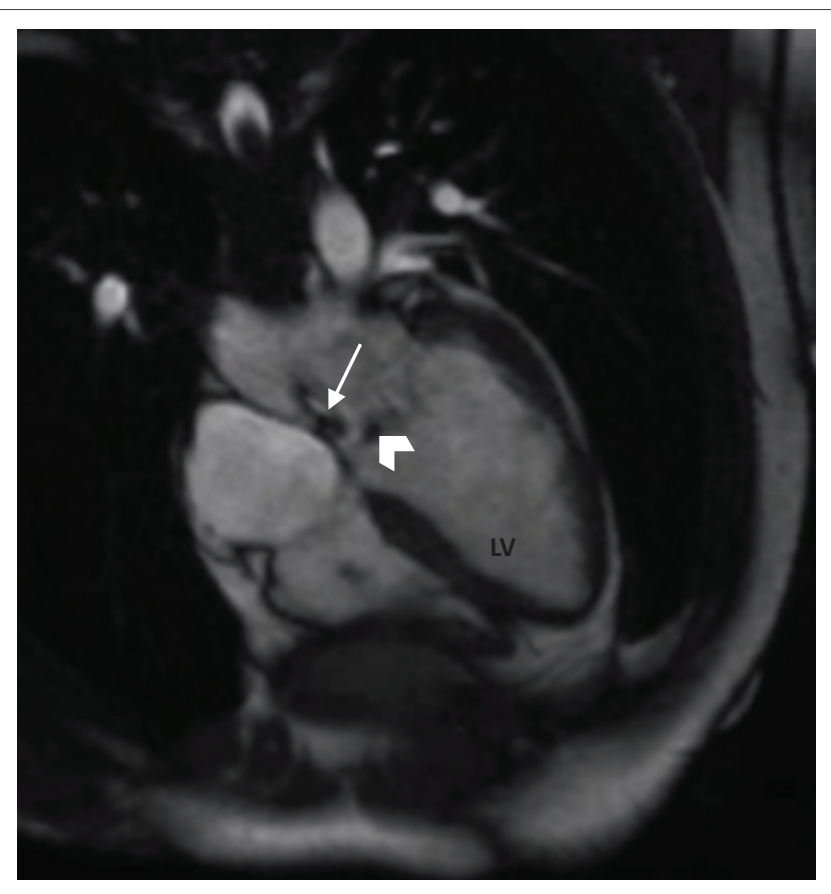

LV, left ventricle.

FIGURE 8: Four-chamber SSFP cine image demonstrates an eccentric flow jet (white arrow) close to the interatrial septum arising from the aortic septum of the mitral valve (arrowhead) owing to a leaflet cleft. Cine image available at https://www.youtube.com/watch?v=eVAduRGblgU\&feature=youtu.be

\section{Papillary muscle and tendinous cords}

There is considerable variation in the number of tendinous cords normally seen attaching to each of the five papillary muscles, with an average of 12 tendinous cords per papillary muscle. The individual papillary muscles are normally the same thickness as the normal left ventricular myocardium.

The most common congenital abnormality of the papillary muscles is a single papillary muscle to which all the tendinous

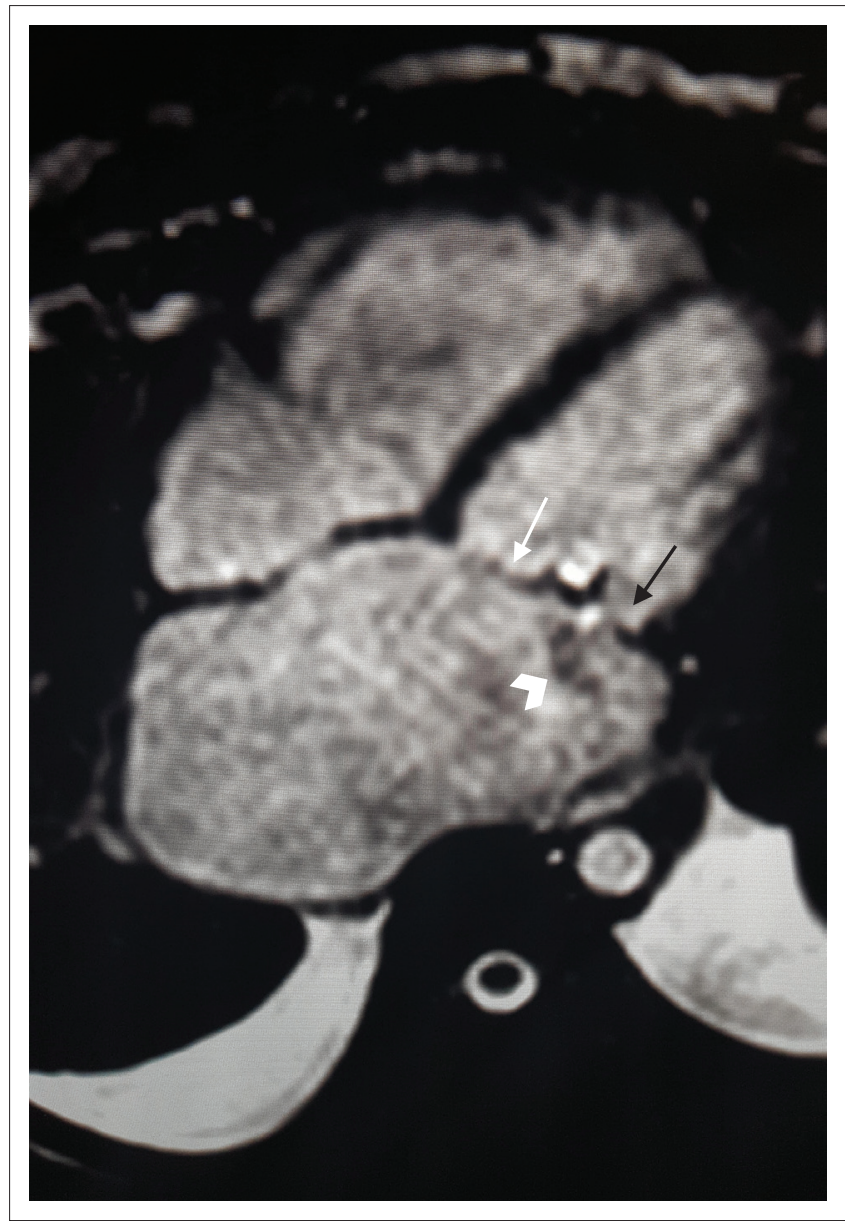

FIGURE 9: SSFP four-chamber view demonstrates prolapse of the anterior leaflet (white arrow) in relation to the posterior leaflet (black arrow) and a posterolateral directed regurgitation jet (arrowhead).

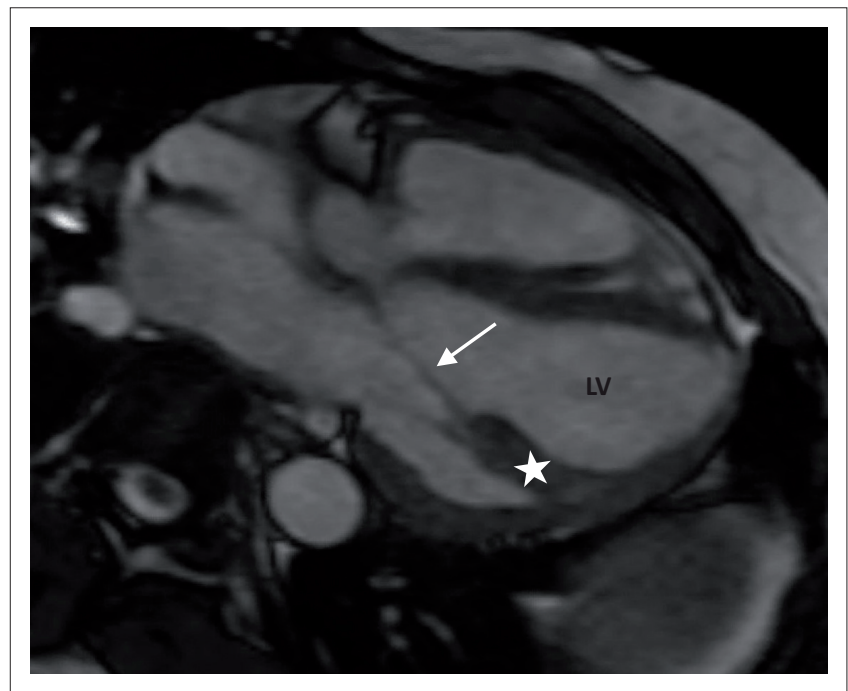

FIGURE 10: Four-chamber cine SSFP image demonstrates a thickened single papillary muscle $\left(^{*}\right)$ in the left ventricle (LV) with all the tendinous cords (white arrow) attaching to this single papillary muscle.

cords of the mitral valve attach (Figure 10), resulting in a 'parachute' mitral valve. ${ }^{9}$

Acquired abnormalities of the papillary muscles are, however, much more common. The most common primary tumour of the papillary muscles is fibroelastoma which may be situated 


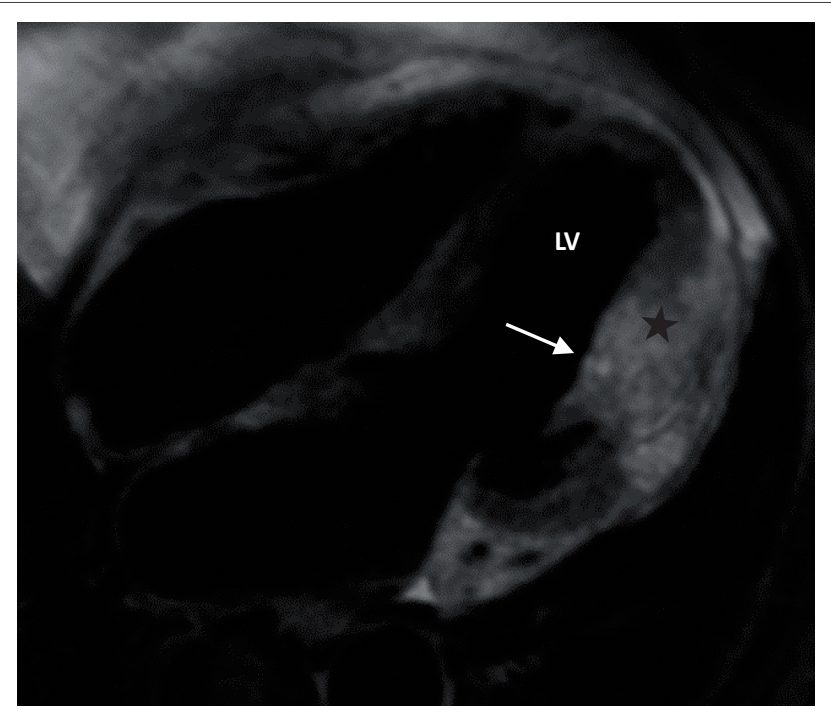

LV, left ventricle.

FIGURE 11: Axial SPAIR image demonstrates extension of a myocardial tumour $(*)$ into the papillary muscle (white arrow).

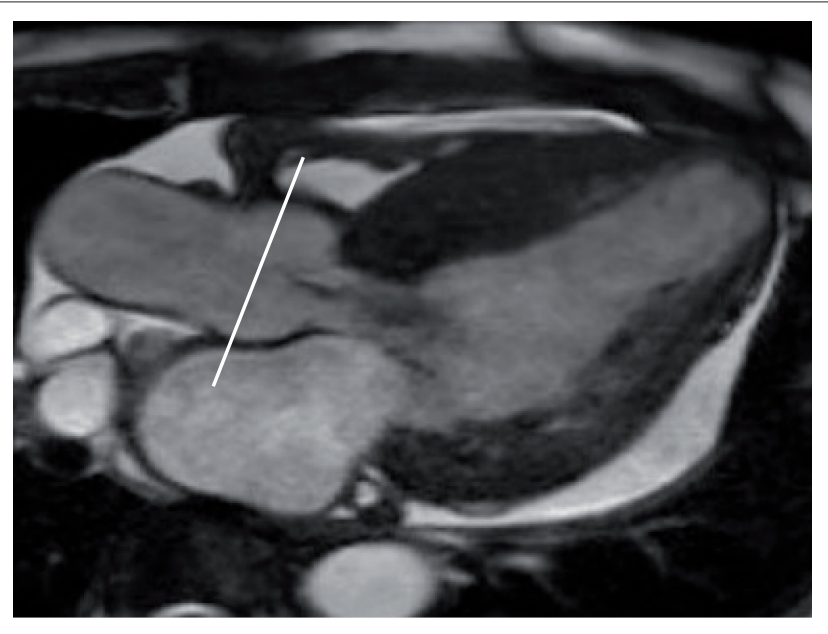

FIGURE 12: LVOT SSFP image demonstrates the orientation of the phase contrast image for the aorta (white line).

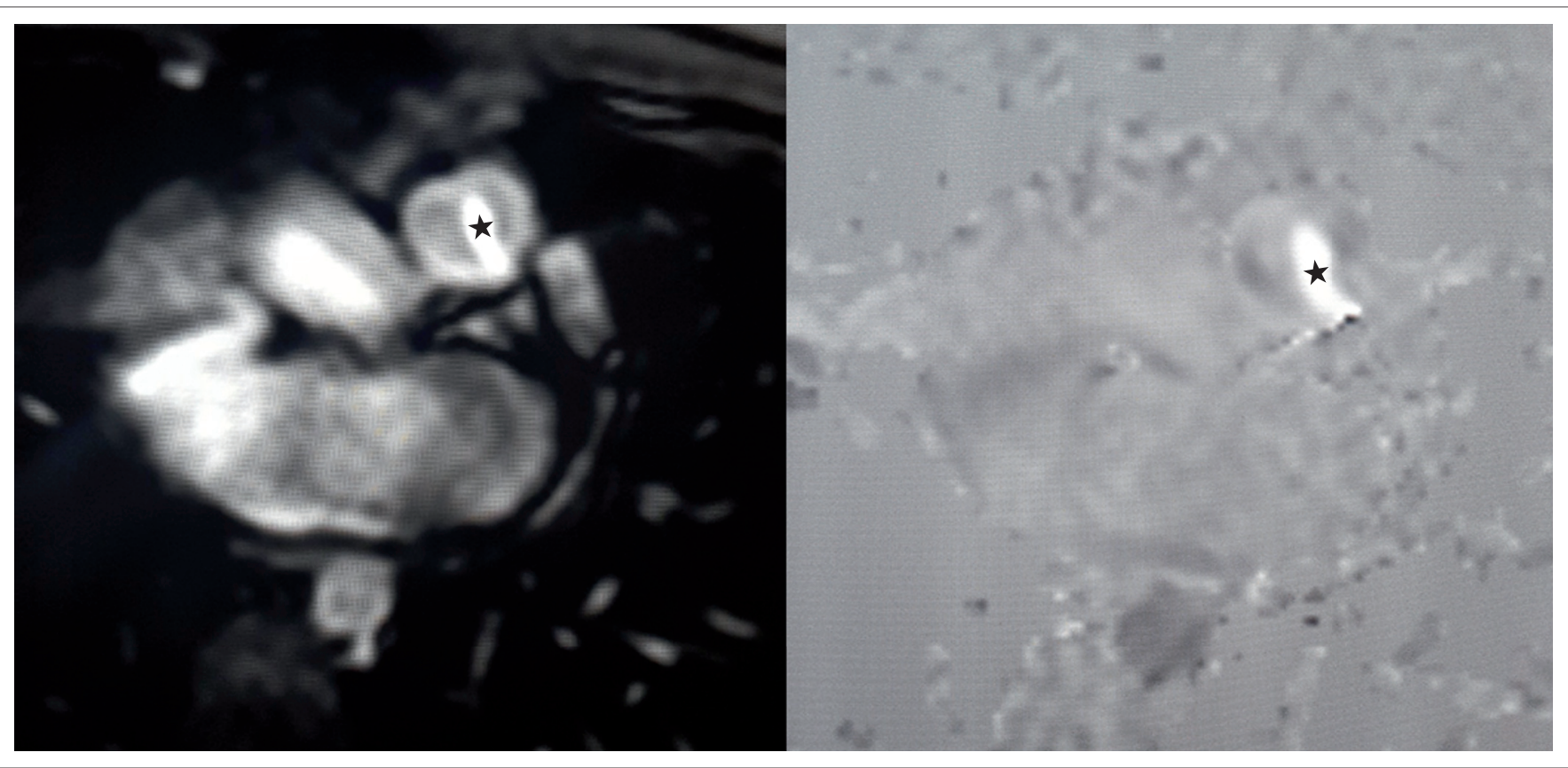

FIGURE 13: Phase contrast images through the pulmonary valve demonstrate pulmonary valve stenosis with flow acceleration (*) demonstrated as increased intensity in the pulmonary artery on the magnitude (left) and phase (right) contrasted images.

on the papillary muscle or on the tendinous cords. However, more common than primary tumours of the papillary muscles is the local invasion or involvement of the papillary muscles owing to direct extension of left ventricular myocardial disease, tumours (Figure 11) or ischaemic scarring.

\section{Valvular function}

SSFP and gradient echo sequences can visualise flow disturbances caused by valvular stenosis or regurgitation on the basis of signal loss owing to dephasing of moving protons. This helps to locate the jet for the dephasing origin and direction. Although SSFP imaging provides better visualisation of valve anatomy, it is less sensitive to phase disturbances than gradient echo sequences, making it easier to miss or underestimate severe regurgitation. ${ }^{10}$

Phase contrast sequences are also used for valvular assessment, particularly of the aortic and pulmonary valves. These sequences are based on the accumulated phase of moving protons and are used to measure the peak velocity, stroke volume, and regurgitation fraction in a vessel. These images needs to be acquired directly perpendicular to the vessel for accurate measurements (Figure 12). Valvular stenosis is seen as increased signal in the region of the flow jet (Figure 13).

\section{Stenosis}

In patients with valvular stenosis, dephasing or a flow jet can be seen distal to the valve (Figure 14). Smaller openings, 


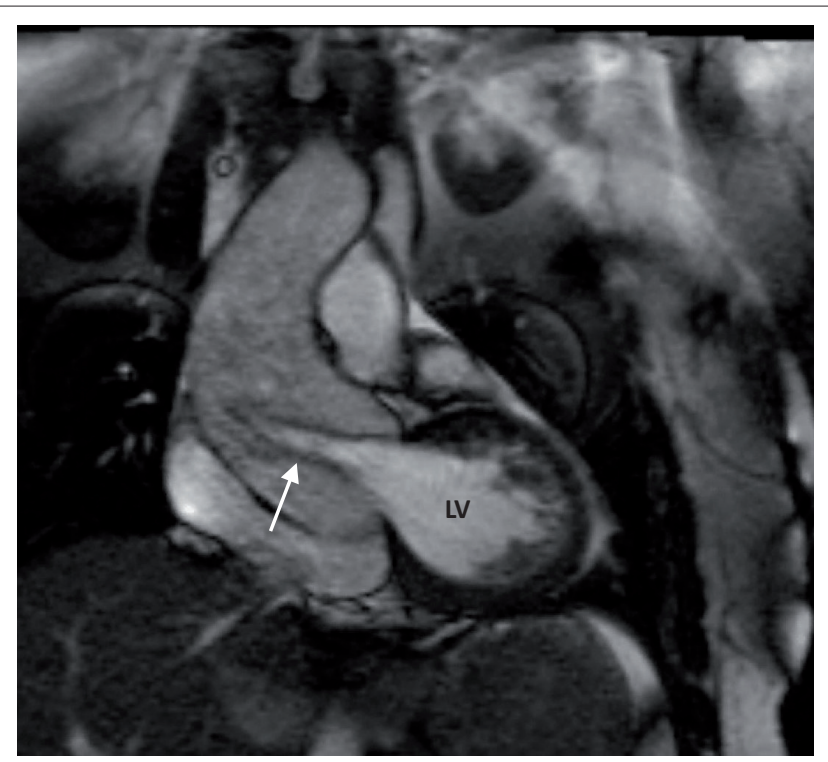

LV, left ventricle.

FIGURE 14: Coronal cine SSFP image of the aortic valve demonstrates a jet (white arrow) distal to the valve as a result of aortic valve stenosis.

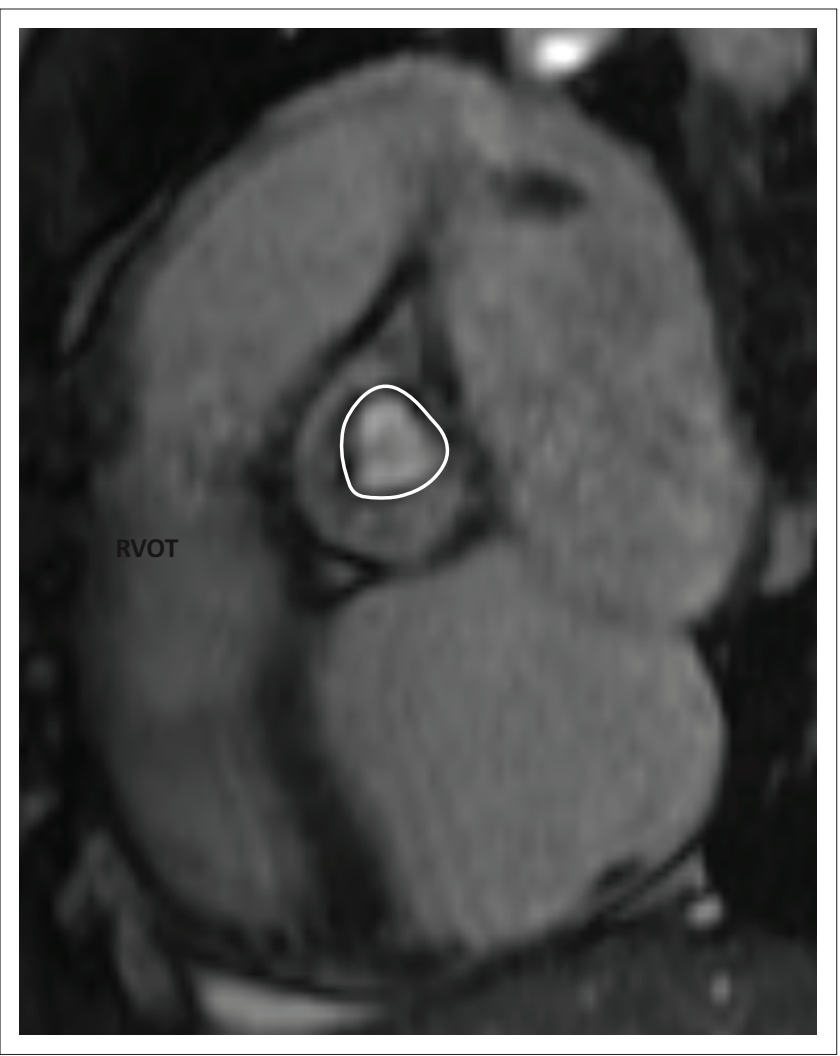

RVOT, right ventricular outflow tract.

FIGURE 15: Axial cine SSFP image of the aortic valve demonstrates maximum opening of the valve traced (white line) to allow for surface area measurement.

characteristic of severe disease, cause more dephasing and a darker jet is seen. The phase contrast images in severe stenosis will also demonstrate higher velocities, which will be seen as an area of complete signal loss owing to aliasing if it is above the programmed maximum velocity. This will result in an underestimation of the peak velocity as velocities higher than the programmed maximum velocities cannot be detected. In these instances, the programmed maximum velocity needs to

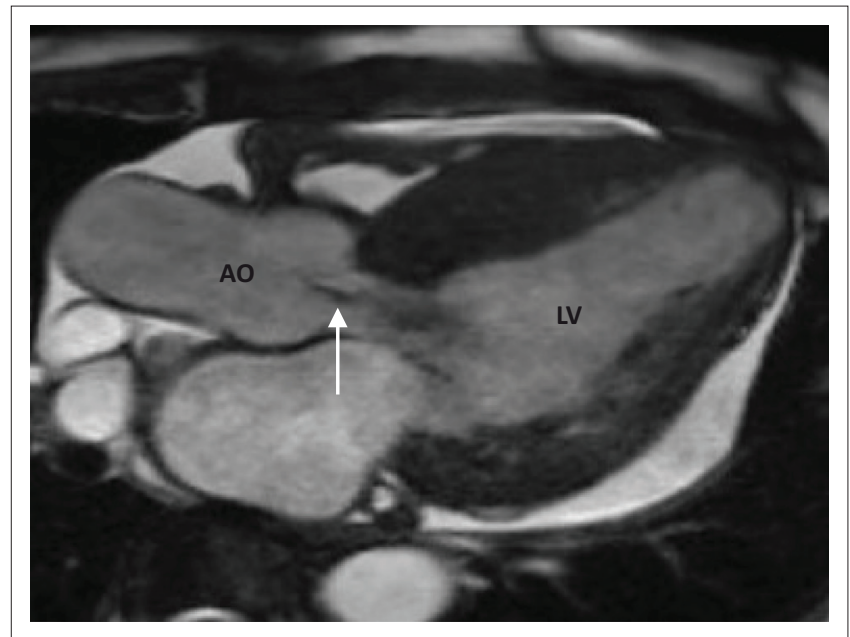

Ao, aorta; LV, left ventricle.

FIGURE 16: LVOT cine SSFP image demonstrates a flow jet (white arrow) proximal to the aortic valve, in keeping with aortic regurgitation.

be adjusted until the aliasing artefact disappears. The area of maximum opening of the valve can also be measured on the axial SSFP cine images through the valve (Figure 15).

\section{Regurgitation}

Valvular regurgitation is seen as dephasing or as a flow jet proximal to the valve (Figure 16). With regurgitation, more severe disease results in a large opening which causes less dephasing and thus results in a less obvious jet.

Aortic and pulmonary valve regurgitation is normally measured directly with phase contrast images. By drawing a region of interest in the vessels, the forward volume and regurgitation volume can be measured and used to calculate the regurgitation fraction (regurgitation volume/forward volume $\times 100){ }^{11}$

Normally, phase contrast images are not used to directly assess the regurgitation fraction for the mitral and tricuspid valves. For these two valves, the regurgitation fraction, as measured on the phase contrast images of the aorta and pulmonary arteries, is calculated using the ventricular stroke volume and the aortic and pulmonary forward volumes respectively:

- mitral regurgitation = left ventricular stroke volume aortic forward stroke volume ${ }^{10}$

- $\quad$ tricuspid regurgitation = right ventricular stoke volume pulmonary forward stroke volume. ${ }^{10}$

\section{Ventricular function}

CMR is not only an excellent modality for assessing the cause of valvular disease and quantifying the severity of the disease, but it has also been shown to be the most reliable modality to assess the consequences of the VHD. ${ }^{12}$ The consequences of VHD are assessed by looking at the ventricular size and function. Because of the reproducibility of CMR, it now forms an integral part in the assessment of these patients for valve replacement surgery. ${ }^{12}$ 


\section{Conclusion}

CMR plays in increasingly important role in the pre-surgical assessment of patients with VHD. CMR is particularly useful to assess valvular anatomy and function, quantify the severity of the disease, as well as assess the sequelae of VHD, with accurate assessment of ventricular size and function.

\section{Competing interests}

The author declares that she has no financial or personal relationships which may have inappropriately influenced her in writing this article.

\section{References}

1. Lang RM, Tsang W, Weinert L, Mor-Avi V, Chandra S. Valvular heart disease. The value of 3-dimensional echocardiography. J Am Coll Cardiol. 2011;58(19): 1933-1944. http://dx.doi.org/10.1016/j.jacc.2011.07.035

2. Hendel RC, Patel MR, Kramer CM, et al. ACCF/ACR/SCCT/SCMR/ASNC/NASCI/SCAI/SIR 2006 appropriateness criteria for cardiac computed tomography and cardiac magnetic resonance imaging: a report of the American College of Cardiology Foundation Quality Strategic Directions Committee Appropriateness Criteria Working Group, Quality Strategic Directions Committee Appropriateness Criteria Working Group,
American College of Radiology, Society of Cardiovascular Computed Tomography, American College of Radiology, Society of Cardiovascular Computed Tomography, Society for Cardiovascular Magnetic Resonance, American Society of Nuclea Cardiology, North American Society for Cardiac Imaging, Society for Cardiovascular
Angiography and Interventions, and Society of Interventional Radiology. J Am Coll Cardiol. 2006;48(7):1475-1497. http://dx.doi.org/10.1016/j.jacc.2006.07.003
3. Bellenger NG, Burgess MI, Ray SG, et al. Comparison of left ventricular ejection fraction and volumes in heart failure by echocardiography, radionuclide ventriculography and cardiovascular magnetic resonance; are they interchangeable? Eur Heart J.2000:21(16):1387-1396. http://dx.doi.org/10.1053/ euhj.2000.2011

4. Wassmuth R, von Knobelsdorff-Brenkenhoff F, Gruettner H, Utz W, Schulz-Menger J. Cardiac magnetic resonance imaging of congenital bicuspid aortic valves and associated aortic pathologies in adults. Eur Heart J Cardiovasc Imaging. 2014;15(6):673-679. http://dx.doi.org/10.1093/ehjci/jet275

5. Xanthos T, Dalivigkas I, Ekmektzoglou KA. Anatomic variations of the cardiac valves and papillary muscles of the right heart. Ital J Anat Embryol. 2011;116(2):111-126.

6. Kim MJ, Yu JJ, Kang SY, et al. Comparison of the offset distance of the tricuspid septal leaflet in neonates with Ebstein's anomaly and neonates with pulmonary atresia with intact ventricular septum. CardiolYung. 2015,25(1):129-136. http:/ dx.doi.org/10.1017/S1047951114001206

7. Delling FN, Kang LL, Yeon SB, et al. CMR predictors of mitral regurgitation in mitral valve prolapse. JACC Cardiovasc Imaging. 2010;3(10):1037-1045. http://dx.doi. org/10.1016/j.jcmg.2010.06.016

8. Wilcox I, Fletcher PJ, Bailey BP. Colour Doppler echocardiographic assessment of regurgitant flow in mitral valve prolapse. Eur Heart J. 1989;10(10):872-879.

9. Schaverien MV, Freedom RM, McCrindle BW. Independent factors associated with outcomes of parachute mitral valve in 84 patients. Circulation. 2004;109(19):23092313. http://dx.doi.org/10.1161/01.CIR.0000129269.98460.C8

10. Cawley PJ, Maki JH, Otto CM. Cardiovascular magnetic resonance imaging for valvular heart disease: technique and validation. Circulation. 2009;119(3): 468-478. http://dx.doi.org/10.1161/CIRCULATIONAHA.107.742486

11. Myerson SG, d'Arcy J, Mohiaddin R, et al. Aortic regurgitation quantification using cardiovascular magnetic resonance: association with clinical outcome. Circulation 2012;126(12):1452-1460.http://dx.doi.org/10.1161/CIRCULATIONAHA.111.083600

12. Lopez-Mattei JC, Shah DJ. The role of cardiac magnetic resonance in valvular heart disease. Methodist DeBakey Cardiovasc J. 2013;9(3):142-148. http://dx.doi. org/10.14797/mdcj-9-3-142 